\title{
Hospital admission and readmission for acute exacerbation of COPD. A tough nut to crack
}

\author{
Michael Steiner ${ }^{1,2}$
}

Reducing acute hospital care for people with long-term conditions has become a key element of health policy as governments strain every sinew to contain the escalating healthcare costs arising from ageing populations. Attention has been drawn to the problem of unscheduled hospitalisation for acute exacerbations of COPD (AECOPD) because it is such a common cause of emergency admission and because there is a high readmission rate following discharge. The latter has driven the perception in many quarters that admissions to hospital should be preventable and, with regards to readmission particularly, may be due to poor quality hospital care. Clinicians are frequently caught in the crossfire of these arguments - under pressure to discharge patients quickly to free up precious hospital bed capacity but also to ensure 'safe' discharge to reduce the risk of further presentation. Primary care physicians are experiencing similar pressures to avoid referral to hospital while at the same time providing safe management of the episode in the patient's home.

The impact of an AECOPD on an individual is determined by the composite effects of the severity of the acute event (eg, respiratory infection) and the underlying condition of the patient (the respiratory disease and other factors such as multimorbidity). In making clinical judgements about discharge from hospital clinicians need to also take into account the social and psychological circumstances, particularly the confidence of the patient and their carers that they are ready to leave hospital. It would be fair to say that currently, these judgements are largely subjective and do not routinely incorporate objective measurements of the risk of subsequent readmission to assist in shared decision-making.

\footnotetext{
${ }^{1}$ Leicester Respiratory Biomedical Research Unit, Centre for Exercise and Rehabilitation Science, Leicester, UK; ${ }^{2}$ School of Sport Exercise and Health Sciences, Loughborough University

Correspondence to Professor Michael Steiner, Leicester Respiratory Biomedical Research Unit, Glenfield Hospital, Groby Road, Leicester LE3 9QP, UK;
} michael.steiner@uhl-tr.nhs.uk
Two papers published in this issue of Thorax are relevant to this debate and have the potential to impact on clinical care. Suh et $a l^{1}$ used parasternal electromyography (EMG) to measure neural respiratory drive (NRD), a surrogate measure of the load imposed on the respiratory system during hospitalisation for AECOPD. Patients whose $\mathrm{EMG}_{\text {para\% }}$ max (parasternal EMG activity during tidal breathing as a proportion of activity during a maximal sniff manoeuvre) did not fall by 3.1 absolute percentage points were at greater risk of short-term readmission (14 days) suggesting that in these individuals, adequate recovery had not yet occurred. This prediction was superior to other simple assessments such as Medical Research Council (MRC) Breathlessness score, Borg breathlessness score and respiratory rate. Indeed the negative predictive power for readmission in patients where NRD did drop by this amount was in the order of $97 \%$. The measurement performed less well as a predictor of readmission at 28 days only doing so in patients aged less than 85 years in a post hoc analysis.

In a study of similar design, Kon et al, ${ }^{2}$ performed $4 \mathrm{~m}$ gait speed (4MGS) at discharge in a cohort of patients hospitalised for AECOPD. This simple measurement of the speed an individual walks over a short $4 \mathrm{~m}$ course is easy to perform in hospital and is widely used in geriatric medicine as a measure of 'frailty' and a risk stratifier for future health outcomes including hospitalisation. The authors observed higher readmission rates at 90 days in patients recording lower quartiles of 4MGS although the measurement was only independently predictive of readmission in patients over the age of 65 years.

These measurements are by no means the first attempts to identify discriminatory biomarkers for future outcomes following AECOPD and hospital admission in particular. Some would appear to relate to the severity or character of the triggering illness (eg, the blood eosinophil count ${ }^{3}$ or Dyspnoea, Eosinopenia, Consolidation, Acidaemia and atrial Fibrillation (DECAF) score $^{4}$ ) and will probably be most useful in predicting short-term outcomes (such as hospital mortality or length of stay) or treatment responses. NRD falls into this category with potential to assist with discharge decision-making. Other indicators such as habitual physical activity ${ }^{5}$ and bedside measures of muscle mass ${ }^{6}$ have been shown to be predictive of hospital readmission after discharge over a longer period (eg, 12 months) and may therefore be indicators of the underlying general health of the patient. The performance of these measures will be affected by the acuity of the event if they are performed before discharge, particularly if they require the cooperation and effort of the patient. It is notable for example that in the study by Greening et $a l^{6}$ rectus femoris cross-sectional area measured by ultrasound (a measure independent of patient effort) was predictive of 12-month hospital readmission whereas maximum voluntary quadriceps strength was not. 4MGS might provide a sensible compromise-a measure that assesses frailty which is easy for staff and patients to perform which appears to retain discriminatory power when performed prior to discharge.

While the studies of Suh and Kon highlight the potential utility of such biomarkers, we need multicentre studies to determine whether these or other measures, when provided to clinicians in the field actually improve decision-making. Standardisation of such measures may not be straightforward. For example the threshold for a significant reduction of NRD of $3.1 \%$ was lower than the standard deviation of the initial measurement. Distinguishing genuine change from measurement variation might therefore confound interpretation of the results if rolled out more widely.

Being able to predict future events does not mean they can be prevented. Evidence from the recent UK national COPD audit ${ }^{7}$ indicates that hospital mortality and length of stay are reducing but hospitalisation for AECOPD remains a stubbornly prevalent problem. Interventions to identify and treat events earlier following their onset such as telemonitoring $^{8}$ or self-management plans ${ }^{9}{ }^{10}$ have generally failed to reduce hospitalisation rates despite often resulting in more treatment provision in the form of systemic corticosteroids and antibiotics. Phenotype specific exacerbation management, for example using the blood eosinophil count as a biomarker, hold promise not least to reduce harm and waste from unnecessary treatment but it is unknown whether this will impact on admission rates. ${ }^{3}$ In broad terms the available evidence suggests that once the acute event is underway, admission (if it is needed) is hard to 
prevent. A more promising approach is to implement prevention strategies in the stable state or in the aftermath of the admission. The most effective intervention in this category is pulmonary rehabilitation (PR) which, when provided within 4 weeks of discharge has been shown to reduce re-hospitalisation in the short to medium term (up to 3 months). ${ }^{11}$ However, enthusiasm for the widespread provision of post-discharge PR has been tempered by clinical experience which suggests few patients who are offered therapy at the point of discharge are willing or able to attend PR in this time frame. ${ }^{12}$ It appears also that comprehensive treatment of sufficient intensity is needed to prevent readmission because a recent trial of a less intensive rehabilitation intervention initiated in hospital and continued in the patient's home was ineffective. ${ }^{13}$ Debate continues about whether it would be better to defer the intervention until the patient has fully recovered and may feel better equipped to undertake conventional PR. While the notion of enhanced recovery through effective rehabilitation is intuitive, so too is the need for the patient to feel they have recovered from the acute event before embarking on a programme of therapy which is demanding on their time and effort. Perhaps an additional utility of a measurement of recovery such as NRD might be to identify when the right time for an individual patient has arrived.

It is notable that in the studies by Suh and Kon as in others, prior admission frequency was an important predictor of readmission. Population studies have suggested that admission frequency may follow an accelerating pattern in some individuals $^{14}$ and although this cannot necessarily be applied to an individual patient, ${ }^{15}$ this pattern may herald the terminal phase of the illness. Expecting a prevention strategy such as PR to prevent further admissions in this context (where proactive palliative care and advance care planning would be more appropriate) is unrealistic. Given the difficulties with prognostication in this population, the extension of biomarkers such as 4MGS from predictors of outcome to prediction of treatment response (eg, to PR) would be a significant advance in refining referral decisions.
Perhaps the key to an effective health systems response to the need for acute admission is ensuring commissioners and policy makers understand the place of these events in the natural history of the condition. In a properly organised and integrated healthcare system where longterm conditions such as COPD are proactively identified and managed, acute crises would be anticipated as part of a managed care programme. AECOPDs requiring hospital care would therefore be considered as 'planned but unscheduled' (we know they are going to happen but we don't know when). This is not to suggest efforts should not be made to prevent hospitalisation. However considering the admission in this way might help ensure the event itself prompts the development of an individualised prevention strategy that can dovetail with wider health promotion measures that are known to have better long-term efficacy in reducing healthcare utilisation such as smoking cessation, physical activity and rehabilitation and activation/engagement of the patient through supported self-management. Incorporating risk stratification biomarkers such as those described in the Journal into routine clinical assessments may have an important role to play in developing such tailored management plans and cracking this particularly hard-shelled nut.

\section{Competing interests None declared.}

Provenance and peer review Commissioned; internally peer reviewed.

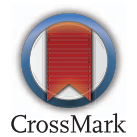

To cite Steiner M. Thorax 2015;70:1108-1109.

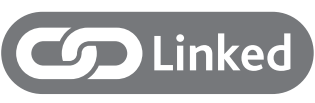

http://dx.doi.org/10.1136/thoraxjnl-2015-207188

- http://dx.doi.org/10.1136/thoraxjnl-2015-207046

Thorax 2015;70:1108-1109.

doi:10.1136/thoraxjn-2015-207986

\section{REFERENCES}

1 Suh ES, Mandal S, Harding R, et al. Neural respiratory drive predicts clinical deterioration and safe discharge in exacerbations of COPD. Thorax 2015;70:1123-30.

2 Kon SSC, Jones SE, Schofield SJ, et al. Gait speed and readmission following hospitalisation for acute exacerbations of COPD: a prospective study. Thorax 2015;70:1131-37.

3 Bafadhel M, McKenna S, Terry S, et al. Blood eosinophils to direct corticosteroid treatment of exacerbations of chronic obstructive pulmonary disease: a randomized placebo-controlled trial. Am J Respir Crit Care Med 2012;186:48-55.

4 Steer J, Gibson J, Bourke SC. The DECAF Score: predicting hospital mortality in exacerbations of chronic obstructive pulmonary disease. Thorax 2012;67:970-6.

5 Garcia-Aymerich J, Lange P, Benet M, et al. Regular physical activity reduces hospital admission and mortality in chronic obstructive pulmonary disease: a population based cohort study. Thorax 2006;61:772-8.

6 Greening NJ, Harvey-Dunstan TC, Chaplin EJ, et al. Bedside assessment of quadriceps muscle by ultrasound after admission for acute exacerbations of chronic respiratory disease. Am J Respir Crit Care Med 2015;192:810-16.

7 Stone RA, Holzhauer-Barrie J, Lowe D, et al. COPD: Who cares matters. National Chronic Obstructive Pulmonary Disease (COPD) Audit Programme: Clinical audit of COPD exacerbations admitted to acute units in England and Wales 2014. London: Royal College of Physicians, 2015.

8 Pinnock H, Hanley J, McCloughan L, et al. Effectiveness of telemonitoring integrated into existing clinical services on hospital admission for exacerbation of chronic obstructive pulmonary disease: researcher blind, multicentre, randomised controlled trial. BMJ 2013;347:f6070.

9 Harrison SL, Janaudis-Ferreira T, Brooks D, et al. Self-management following an acute exacerbation of COPD: a systematic review. Chest 2015;147: 646-61.

10 Bucknall CE, Miller G, Lloyd SM, et al. Glasgow supported self-management trial (GSUST) for patients with moderate to severe COPD: randomised controlled trial. BMJ 2012;344:e1060.

11 Puhan MA, Gimeno-Santos E, Scharplatz M, et al. Pulmonary rehabilitation following exacerbations of chronic obstructive pulmonary disease. Cochrane Database Syst Rev 2011;(10):CD005305.

12 Jones SE, Green SA, Clark AL, et al. Pulmonary rehabilitation following hospitalisation for acute exacerbation of COPD: referrals, uptake and adherence. Thorax 2014;69:181-2.

13 Greening NJ, Williams JEA, Hussain SF, et al. An early rehabilitation intervention to enhance recovery during hospital admission for an exacerbation of chronic respiratory disease: randomised controlled trial. BMJ 2014;349:g4315.

14 Suissa S, Dell'Aniello S, Ernst P. Long-term natural history of chronic obstructive pulmonary disease: severe exacerbations and mortality. Thorax 2012;67:957-63.

15 Sadatsafavi M, FitzGerald JM. Heterogeneity's ruses: the neglected role of between-individual variability in longitudinal studies of COPD exacerbations. Thorax 2014;69:1043-4. 